\section{Beteiligung lernen}

Diskussionsbeitrag anlässlich des

„Bürgerdialogs Standortsuche“ der Endlager-Kommission

von Yasmine Kühl, Institut für Technikfolgenabschätzung und Systemanalyse (ITAS), Karlsruhe, Felix Wittstock, Friedrich-Schiller-Universität Jena, und Nele Wulf, ITAS, Karlsruhe

Partizipation stellt im Bereich der Technologiepolitik nach wie vor einen Trend und im Fall der Endlager-Kommission ein „Pionierverfahren" dar. Exemplarisch für die Debatte um die Sinnhaftigkeit und Angreifbarkeit professionell organisierter Bürgerbeteiligung geriet beim "Bürgerdialog Standortsuche“ neben Fragen sachgerechter Problembearbeitung und Verfahrensgerechtigkeit auch die Ebene der Medialisierung in den Blick. Vor diesem Hintergrund wäre Partizipation zunächst als Untersuchungsgegenstand per se und nicht als Lösungsstrategie zu fassen. Die methodische Auseinandersetzung in dieser Frage weist weit über einzelne Veranstaltungen und die Endlagerthematik hinaus.

Partizipative Verfahren versprechen Vertrauen und erhöhte Akzeptanz für weitreichende technologiepolitische Maßnahmen. Ein entsprechendes Partizipationsverfahren fand am 20. Juni 2015 in Berlin statt: Die Kommission „Lagerung hoch radioaktiver Abfallstoffe“ initiierte den „Bürgerdialog Standortsuche“ (Tagungsbericht Kühl et al. 2015). Ausführende Veranstalter waren DEMOS (Beteiligungskonzept) und Prognos (Moderation). Dieser Bürgerdialog verdient in mehrfacher Hinsicht besondere Beachtung, u. a. entspricht er explizit nicht den von Alexander Bogner (2010) für die New \& Emerging Science and Technology (NEST) herausgearbeiteten Beobachtungen einer weitgehend experimentellen Laienbeteiligung, die sich ohne Bezugnahme auf öffentliche Kontroversen, politische Teilhabebestrebungen sowie individuelle Betroffenheit vollziehe.

Im Gegenteil: neben den hochpolitischen Konflikt um die Nutzung der Atomenergie tritt die nun dringend nach Antworten verlangende Frage der Entsorgung. Die enorme Herausforde- rung, ein angemessenes Verfahren auf dem Weg zu sachgerechter und demokratisch legitimierter Lagerung hoch radioaktiver Abfallstoffe zu finden, verbindet sich vor dem Hintergrund der jahrzehntelangen Konfliktgeschichte (Grunwald 2016) mit hohen Erwartungen an die Problemlösungsfähigkeit einer modernen Gesellschaft und ihrer politischen Institutionen. Die Akzeptanz des Verfahrens ist grundlegend für die Legitimation eines später zu bestimmenden Endlagerstandortes. Diese normative Dimension lässt sich um die Betroffenheit zukünftiger Generationen sowie Aspekte der Nachhaltigkeit und Gerechtigkeit ergänzen (Grunwald 2015). Öffentlichkeitsbeteiligung in der Endlagerdebatte bedeutet daher - in einem bislang kaum gekannten Maße - eine Auseinandersetzung mit Fragen der Demokratietheorie, angewandter Beteiligungsmethodik und Transparenz. Zahlreiche dieser Aspekte kamen im Vorfeld des Bürgerdialogs auch in den Erwartungen der Kommission zum Ausdruck. In diesem Beitrag vergleichen wir die formulierten Ansprüche der Kommission mit unseren Beobachtungen. Um ein umfassendes Bild zu erhalten, haben wir Gespräche mit Teilnehmern und Kommissionsmitgliedern geführt sowie die Presseberichterstattung und den Evaluationsbericht der Kommission (2015a) einbezogen. Abschließend diskutieren wir den Bürgerdialog im Kontext von Medienwissenschaften, Partizipations- und Technikakzeptanzforschung sowie Demokratietheorie.

\section{Das Partizipations-Transparenz-Dilemma}

Essentieller Bestandteil eines jeden Partizipationsverfahrens sind interessierte und engagierte Teilnehmer ${ }^{1}$. Der Befürchtung der Initiatoren ${ }^{2}$ des „Bürgerdialogs Standortsuche“, womöglich zu wenige Interessenten zu finden, wurde in Einladungs- und Anmeldepraxis jedoch nur eingeschränkt entsprochen: Die Anmeldung erfolgte ausschließlich online über eine Webseite von XING Events. Teilnehmer mussten hierbei die AGBs des Seitenbetreibers bestätigen und eine Datennutzungsvereinbarung mit dem OnlineNetzwerk eingehen, das in keinem weiteren $\mathrm{Zu}$ sammenhang mit dem Thema der Veranstaltung stand und von Datenschützern kritisiert wird. Die 
„Nutzerverwaltung“ über ein soziales Netzwerk ist gerade in der Antiatomkraftszene bedenklich, wo auf Datenschutz sensibel geachtet wird.

Es ist umstritten, inwiefern sich der als gesellschaftliche Forderung an politische Entscheidungsverfahren gerichtete Transparenzanspruch auch auf Bürger beziehen soll, die partizipieren wollen (,Partizipations-Transparenz-Dilemma", Seemann 2011 am Bsp. der Piraten-Konsultationsplattform Liquid Feedback; zu Auswirkung von real name policy auf die Teilnahmebereitschaft an Online-Beteiligung exemplarisch Ruesch/Märker 2012). Anschlussfähig ist, dass der Anspruch auf Mitbestimmung dort im Widerspruch zur Forderung nach Privatheit steht, wo er sich auf die notwendige Nachvollziehbarkeit inhaltlicher Vorschläge bezieht, d. h. Manipulationen möglich sind.

Der Bürgerdialog hat deutlich gemacht, dass bei zukünftiger Präsenzbeteiligung trennschärfer zwischen der Transparenz von Prozessen und Ergebnissen (s. u.) und dem Schutz personenbezogener Daten (,gläserner Bürger") unterschieden werden sollte. Die beim Bürgerdialog gewählte Form ermöglicht es Mitgliedern des Internetportals, sich ein Bild über die Teilnehmer zu verschaffen. Auch für Nichtmitglieder des Netzwerks entstand so ein Profil mit Klarnamen und Institution, das der weiterhin einsehbaren Gästeliste der Veranstaltung zugeführt wurde. Dieser Panoptikumseffekt führte dazu, dass Angemeldete im Vorfeld Kontaktanfragen anderer erhielten und weiterhin erhalten. In der Anmeldephase von Präsenzbeteiligung und kombinierten Verfahren mit zusätzlicher Onlinephase wäre es grundsätzlich möglich, eine Zuordnung der Teilnehmer als nicht intendierte Nebenfolge zu vermeiden. Eine Anwendung des Datenschutzkonzepts „PIA“ (Privacy-Impact-Assessment, De Hert et al. 2012) wäre hier denkbar.

\section{Die Medialisierung von Partizipation}

Aus Gründen der Transparenz, Verwertbarkeit und Nachvollziehbarkeit ist die Dokumentation von Bürgerbeteiligung notwendig. Bei Präsenzveranstaltungen möchten wir fünf Dimensionen der Medialisierung voneinander unterscheiden:
1. Öffentlichkeitsarbeit: Dokumentation der Veranstaltung zum Zweck der Berichterstattung (z. B. Fotos, Videos)

2. Ergebnissicherung: Dokumentation der Ergebnisse innerhalb der laufenden Veranstaltung (z. B. Bürgervorschlag/Graphic Visualisation auf Stellwänden durch Moderatoren oder Teilnehmer), methodenabhängig auch zur Weitergabe an wechselnde Diskussionsgruppen

3. Ergebniskommunikation: innerhalb der laufenden Veranstaltung (z. B. mündliche $\mathrm{Zu}$ sammenfassungen durch Moderatoren oder Teilnehmer), zur Weitergabe an wechselnde Diskussionsgruppen oder als Abschlusspräsentation im Plenum

4. Ergebnisveröffentlichung: gemeinsame Stellungnahme (z. B. Ergebnispapier, Bürgerreport)

5. Evaluation: Dokumentation der Diskussionen selbst (z. B. Gesprächsprotokolle, Mitschnitte von Kleingruppendiskussionen, Fragebögen, Leitfadeninterviews mit Teilnehmern)

Die Dimensionen bedürfen sorgfältiger Unterscheidung und können in ein Spannungsverhältnis zwischen neutraler Vermittlung und AgendaSetting geraten. Sie sollten den Teilnehmern daher im Vorfeld erläutert oder je nach Anlass zur Diskussion gestellt werden, da sie potenziell in Konflikt miteinander treten und so das übergeordnete Beteiligungsziel der Vertrauensbildung gefährden können.

\section{Dialog versus Öffentlichkeitsarbeit}

Die auf Öffentlichkeitsarbeit zielende Dokumentation von Beteiligung kann akteursübergreifend politisch legitimierend eingesetzt werden. Dieser Vorwurf äußerte sich beim Bürgerdialog in dem von Teilen der Umweltbewegung artikulierten Misstrauen, durch ihre Teilnahmebereitschaft als „Statisten“ in einer Akzeptanz schaffenden medialen Verwertung des Verfahrens instrumentalisiert zu werden (,Embedded Zivilgesellschaft", Becker 2016, S. 178; „Demokratie-Inszenierung“, Kühl et al. 2015, S. 135). Diese Wahrnehmung ist von anderen Beteiligungsanlässen bekannt (Wilk/Sahler 2014; Wagner 2013) und wurde beim Bürgerdialog durch die Präsenz eines offensiv auftretenden Kamerateams eher 
befördert. Mit ihrer Unterschrift gaben die Teilnehmer ihr rechtliches Einverständnis zur Präsenz des vom Veranstalter beauftragten Kamerateams „reframe“ und der späteren Verwendung der Bilder. Die Aktivität des Teams während der Diskussionsphasen wurde von mehreren Teilnehmern als störend und rein illustrativ thematisiert. Es ist anzunehmen, dass sie Einfluss auf die Beteiligung hatte. Medialisierung zum Zwecke der Öffentlichkeitsarbeit tritt hier in Konflikt mit der Bereitstellung des ,geschützten Raumes“, in den Bürger geladen werden, um sich zu äußern. Grundsätzlich wäre im Vorfeld von Präsenzveranstaltungen zu klären, welche Öffentlichkeit beteiligt werden soll - die anwesende und/oder eine durch Berichterstattung vermittelte. In früheren Verfahren hatte eine zu intensive Dokumentation, welche für die Diskussion keinen Mehrwert bot, bereits zu Abbruchandrohungen der Teilnehmer geführt (Bogner 2010, S. 89).

Dass der Bürgerdialog medial vermittelt wird und wie dies geschieht, ist keineswegs trivial. Die so vermittelte Dimension der Welterfahrung spielt bei der Konstruktion der sozialen Wirklichkeit eine große Rolle. In diesem Sinne spricht Kreimeier (2001) von der Medienwirklichkeit, in der mediale Erfahrungen die alltäglichen ergänzen, mit ihnen konkurrieren oder sie unterwandern und somit ,zum festen Inventar des modernen Bewusstseins gehören" (S. 445). Gerade in Bezug auf den zukünftigen Stellenwert dieses und weiterer Verfahren im Standortauswahlprozess ist die Einschätzung relevant, dass die Bedeutung von Repräsentationen diejenige konkreter Ereignisse sogar übersteigen könnte.

Die Ergebnissicherung innerhalb von Partizipationsformaten beinhaltet Herausforderungen, die methodisch reflektiert und in ihrer Komplexität auch offen kommuniziert werden sollten. Zur Disposition stehen die Ausgewogenheit der Beteiligungsergebnisse, deren inhaltliche Verwertbarkeit sowie die Glaubwürdigkeit des Verfahrens selbst. Um Vertrauen in den Prozess nicht zu enttäuschen oder Misstrauen zu schüren, bedarf es dafür gerade bei heiklen Themen einer professionellen Moderation. Der Bürgerdialog bot in dieser Hinsicht ein heterogenes Bild (Kommission 2015a).
Im Rahmen der Formate „World Café“ und „Fokusgruppen“ war eine intensive Ergebniskommunikation vorgesehen. Die Gruppenphasen boten jedoch zu wenig Zeit, um Ergebnisse angemessen zu erarbeiten und zwischen den Gruppen weiterzugeben. $\mathrm{Zu}$ enge Frageschemata führten im World Café zudem dazu, dass rege geführter, fachlicher Dialog nicht dokumentiert wurde, da dieser im vorgegebenen Antwortraster der Stellwand nicht zu verwerten war. An anderer Stelle wurden während der Diskussion Äußerungen von der Moderation schnell als „Konsens“ bezeichnet und vornehmlich Aspekte protokolliert, die mit größerer Intensität vorgetragen wurden. Solche Beobachtungen verweisen auf ein für diese Formate typisches Problem: Das Vorhaben, aus einer vielschichtigen Diskussion Ergebnisse abzuleiten und diese nachvollziehbar darzustellen, ist immer eine Herausforderung und zwangsläufig unvollständig. Dementsprechend muss besonderes Augenmerk darauf gelegt werden, vereinseitigende Darstellungen und vorschnelle Themensetzungen zu verhindern. Auf eine entsprechende Frage aus dem Publikum antwortete eine Mitarbeiterin von Prognos: „Wir lösen das“. Es wurden jedoch keine Hinweise gegeben, wie mit der Herausforderung, Meinungsbildungsprozesse abzubilden, zukünftig umgegangen wird.

Abschlusspräsentationen im Plenum können Gruppenkonsens erlebbar machen und im Fall des Bürgerdialogs crossmedial vermitteln. Umso bemerkenswerter war, dass es in dieser Phase zu ,uneingeladenem Protest" (Böschen/ Walk 2014) aus dem Publikum kam. Einspruch hervorgerufen hatte v. a. die Zusammenfassung eines CDU-Politikers aus dem Gorleben-Landkreis Lüchow-Dannenberg, der die Präsentation der Kleingruppe anstelle des Prognos-Moderators übernommen hatte. Der Politiker „repräsentierte“ damit Teilnehmer der „kritischen Öffentlichkeit" (Kommission 2015b, S. 15), noch dazu Schüler und Lehrer seines Landkreises. Er schilderte, in seiner Diskussionsgruppe seien Tendenzen einer spezifischen „German Angst“ thematisiert worden, einer deutschen Technikfeindlichkeit somit, die nicht entstehen dürfe. Dieses Resümee und seine performative Setzung als Gruppenkonsens wurde von den Teilnehmern seiner Gruppe per Wortmeldung vehement abge- 
lehnt („Reduzierung auf eine griffige Formel“, „wurde so nicht gesagt"). Andere Anwesende unterstellten daraufhin den Diskussionsleitern, absichtlich wichtige Forderungen zu unterschlagen (z. B. die nach einem Bürgerentscheid). Zunehmende Kritik an der Wiedergabe von Diskussionsverläufen setzte die Moderatoren unter Rechtfertigungsdruck und mündete in eine grundlegende Hinterfragung der methodischen Anforderungen an Beteiligungsverfahren - verbunden mit einem wachsenden Bewusstsein für den besonderen Einfluss der Diskussionsleiter. Bereits zuvor war in einer Kleingruppe die Forderung nach unabhängigen Partizipationsbeauftragten aufgekommen.

\section{Aus der Forschung lernen}

Aus Sicht der Technikfolgenabschätzung (TA) ist dieser "Moderationsunfall“ in mehrfacher Hinsicht erwähnenswert, fiel der Vorwurf einer pauschalen deutschen Risikowahrnehmung doch hinter Erkenntnisse als auch Empfehlungen der Technikakzeptanzforschung zurück (u. a. Hennen 1994; Renn/Zwick 1997; acatech 2011; Deutschlandradio Kultur 2015). Stattdessen entsprach die Zuschreibung emotionaler Vorbehalte einer Funktionslogik des Emotionalisierens bzw. Ent-Rationalisierens sachlicher Gegenargumente. Die langjährige Erfahrung der partizipativen TA und die starke Fachausrichtung einer Parallelveranstaltung von Atomkraftgegnern zeigen dagegen, dass Bürger sich den Sachfragen deliberativer Beteiligungsverfahren mit einem hohen $\mathrm{Maß}$ an Rationalität widmen können - und im Gegenzug eine ebenso sachliche Haltung und Ehrlichkeit von den Initiatoren erwarten (Decker/Fleischer 2014). Zudem ist die Relevanz emotionaler Momente zunehmend Teil der theoretischen Auseinandersetzung mit Beteiligung (Rossnagel et al. 2014, S. 332; Kropp 2013).

\section{Unvoreingenommene Profis?}

Wie kann „eingeladene Partizipation“ (wie im Fall der Schüler aus Gorleben) methodisch und fachlich gelingen, ohne in den Verdacht des Agenda-Settings zu geraten? Gleichheits- und Neutra- litätsideale, die als Grundlage eines erfolgreichen Dialogs angesehen werden (Sturgis 2014), stehen in der Praxis einer Ungleichverteilung von Beteiligungskompetenzen, politischen Interessen und fachlicher Expertise gegenüber. Vielleicht zur Bekräftigung des Verfahrensmottos, man wolle „Beteiligung lernen“, wurde beim Bürgerdialog darauf verzichtet, auf den existierenden wissenschaftlichen Diskurs über partizipative Verfahren zu verweisen und diese nur selten als eine seit mehreren Jahrzehnten erprobte Praxis beschrieben. In einer für die Kommissionswebseite gefilmten Abschlussrede hieß es, die Kommission verstehe unter „Lernen“ nicht bekannte dissensfördernde Hierarchien (Fächer, Didaktik, Methodik) oder ein festes Curriculum, auch nicht die Trennung zwischen Lehrern und Schülern, sondern einen Dialogprozess. Diese Aussage legt die vertrauensbildendende Absicht der Kommission dar, in der Konfliktfrage gemeinsam Neuland betreten zu wollen. Machtasymmetrien zugunsten der oftmals nur durch eine Seite beauftragten ,vertrauenswürdigen Dritten“ (Rossnagel et al. 2014, S. 333; Kühl et al. 2015, S. 134) können von den „Beteiligten“ als Hindernis wahrgenommen werden. Doch auch die in der Atompolitik sich mit fachlicher Expertise verbindende Interessengebundenheit (Grunwald et al. 2002, S. 14) erzeugt fast zwangsläufig Widersprüche: Moderatoren wie Teilnehmer, die dem Konfliktgegenstand Atomkraft wie den unterschiedlichen Interessenlagen der beteiligten Stakeholder und deren vorhandenen Machtasymmetrien gerecht würden, könnten demnach kaum allparteilich sein - und unvoreingenommene Diskussionsleiter und Teilnehmer dem Vorwurf nach keine Experten. Wie herausfordernd die Reflexion möglicher Widersprüche in der partizipativen Endlagerstandortsuche ist, ließ sich am Beispiel der Einbindung einer vermeintlich marginalisierten Gruppe miterleben: Während den vonseiten der Initiatoren umworbenen Jugendlichen aus Gorleben (Dehmer 2015) von älteren Mitdiskutanten mitunter abgesprochen wurde, bei der Thematik (Konfliktgeschichte) mitreden zu können, reflektierte einer der Schüler in seiner Diskussionsgruppe unaufgefordert und selbstkritisch seine mögliche Befangenheit und hinterfragte ihre explizite Einladung durch die Initiatoren. Angesichts dieser Komplexitäten eine zustimmungsfähige, 
authentische und legitimitätsstiftende Öffentlichkeitsbeteiligung zu gestalten, scheint uns die größte Herausforderung zu sein.

\section{Emotionsmanagement und mediative Elemente}

Bürgerdialoge und Mediationsverfahren stehen seit einiger Zeit in der Kritik, politische Konflikte mittels bestimmter Kommunikationsstrategien „lösen“ zu wollen. Die Rede ist von „Emotionsmanagement", d. h. von, ,auf Exploration und Expression von Gefühlen abzielenden Techniken“ (Bröckling 2015, S. 185), welche unterschiedliche Interessenlagen psychologisieren. Der beim Bürgerdialog viel zitierte Dissens könnte so auf einen Mangel an Gesprächsbereitschaft oder -kompetenz zurückgeführt werden: „Die Fokussierung auf Face-to-Face-Kommunikation und das über einen unabhängigen Dritten angeleitete Emotionsmanagement nährt die Suggestion, dass Streitfälle sich beilegen lassen, wenn man nur lang genug und in geeigneter Form miteinander redet. Ein Verfahren, das im Wesentlichen aus einem Arrangement klarer Gesprächsregeln und -schritte besteht, tendiert dazu, Konflikte als kommunikative Dysfunktionen zu traktieren.“ (Bröckling 2015, S. 185) Exemplarisch nachvollziehen ließ sich dies am Vorschlag einer Prognos-Moderatorin in ihrer Abschlusspräsentation, für das politisch umstrittene „Endlager“ einfach einen anderen Begriff zu verwenden ${ }^{3}$. In der „Verheißung, Kommunikationsstörungen kommunikativ entstören zu können“, liegt dabei auch eine Schuldzuschreibung: Bleibt eine Einigung aus, wird dies mangelndem kommunikativem Einsatz zugeschrieben. „Sich dem Gespräch zu verweigern oder es abzubrechen, gilt als Sündenfall schlechthin.“(Bröckling 2015, S. 185)

\section{Konsens-Demokratie vs. Konflikt- Demokratie}

Fragen der Partizipation berühren grundlegend das Verständnis von Demokratie. In dieser Hinsicht war das Resümee eines Kommissionsmitglieds aufschlussreich, in welchem er aufforderte, die Funktionsfähigkeit der Demokratie gegenüber anderen Systemen unter Beweis zu stellen. Uns stellt sich jedoch die Frage, wie sich die hier beschriebene Form von Beteiligung zur repräsentativen Demokratie verhält. Wer ist die Öffentlichkeit und welchen Einfluss darf oder soll sie nehmen? Dies sind demokratietheoretische Fragen, welche die Kommission, ebenso wie ein mögliches zukünftiges Gremium ${ }^{4}$, nicht unbeachtet lassen darf, da sie grundlegend für die Legitimation ihrer Arbeit sind. Zusätzlich zur Frage, ob und wie deliberative Elemente in eine repräsentative Demokratie integriert werden sollten, geht es um eine noch basalere Unterscheidung: Konsens oder Konflikt? Wie steht das in vielen Aussagen der Kommission aufscheinende Ideal einer auf Konsens und Deliberation ausgerichteten Demokratie zu einem Konzept von Demokratie, das die antagonistischen Dimensionen des Politischen ernst nimmt? Es gilt, sich grundlegend mit Befürchtungen auseinanderzusetzen, Konflikte könnten gewaltsam aufbrechen, sofern ,keine Kanäle [...] da sind, über die sie eine ,agonistische“ Form annehmen könnten“" (Mouffe 2007, S. 12).

Im Lichte dieser Überlegungen konnte die von Teilen der Umweltbewegung organisierte Parallelveranstaltung „A tommüll ohne Ende, Teil 2 " betrachtet werden. Deren Organisatoren hatten die Teilnahme am Dialog in der von der Kommission initiierten Form abgelehnt. Ein Verhalten, das den deliberativen Idealen zuwiderläuft, aber nicht als weniger demokratisch, geschweige denn als geringer wertig bezeichnet werden kann. Denn ,[a]uch die kompetenteste Anwendung der Regeln sachbezogenen Verhandelns ändert nichts daran, dass die Verhandlungsmacht in der Regel asymmetrisch verteilt ist." (Bröckling 2015, S. 184) Die soziale Ungleichheit wird durch die formale Gleichheit der Vertragsparteien unsichtbar gemacht: Verfahrensteilnahme und Zustimmung zu einer Vereinbarung lassen diese Asymmetrien bestehen und verschaffen ihnen die Legitimation des Konsenses (Bröckling 2015, S. 184). Während die Stärkeren davon profitieren, die Schwächeren vertraglich einzubinden, auch wenn diese ihnen dafür entgegenkommen müssen, können jene ,[...] Zugeständnisse heraushandeln, verzichten dafür aber auf die Option, den Konflikt eskalieren zu lassen und die Kräfteverhältnisse möglicherweise so nachhaltiger zu 
ihren Gunsten zu verschieben." (Bröckling 2015, S. 185) Folgt man dieser Überlegung, haben die Teilnehmer der Parallelveranstaltung sich genau darauf nicht eingelassen. Die Veranstalter des Bürgerdialogs adressierten zwar die im Beteiligungskonzept so bezeichnete ,kritische Öffentlichkeit" (Kommission 2015b, S. 15) im Rahmen ihrer Zielgruppen-Konzeption. Damit bleibt aber die Frage „Welche Demokratie?“ ausgeblendet.

\section{Fazit}

„Beteiligung lernen“- dieses Motto war von der Endlager-Kommission anspruchsvoll gewählt und soll sicher zugleich Prozesshaftigkeit und Veränderungsbereitschaft ausdrücken. Es bedarf unseres Erachtens eines sensiblen Umgangs mit den sich daraus ergebenden Herausforderungen, besonders angesichts der Tragweite des Themas. Methodische Mängel der Partizipationsveranstaltung bezogen sich aus unserer Sicht einerseits auf den organisatorischen Rahmen der Veranstaltung. Zugang, Datenschutz, Bilddokumentation verweisen auf ein Spannungsverhältnis zwischen der Intention breiter Beteiligung und Transparenz, ihrer tatsächlichen Umsetzung und einer auf medialen Erfolg ausgerichteten Aufbereitung. Andererseits lassen sich verschiedene Elemente der inhaltlichen Gestaltung und Durchführung identifizieren, die der Bedeutung der Veranstaltung und den Beteiligungswünschen der Teilnehmer unangemessen waren. Moderation, Ergebnissicherung und die Kommunikation zwischen Veranstaltern und Teilnehmern konnten beim Bürgerdialog oftmals nicht den Ansprüchen an eine sachgerechte, reflektierte und respektvolle Auseinandersetzung mit dem Problem nuklearer Entsorgung entsprechen. Auch müsste für zukünftige Partizipationsveranstaltungen der zeitliche Rahmen im Hinblick auf die formulierten Erwartungen und Ansprüche realistisch gewählt werden. Unsere Beobachtungen können als empirisches Material Eingang in weitere wissenschaftliche Analysen finden. Diese sind angesichts der zahlreichen Herausforderungen, vor die Beteiligungsverfahren gestellt sind, gefordert. Es ist davon auszugehen, dass Partizipationsverfahren auch in Zukunft die Dynamik der Endlagerstandortsuche und anderer Politikfelder beeinflussen werden. Deshalb weist das Ringen um den „besten Weg“ weit über einzelne Veranstaltungen, ihre Stärken und Schwächen und die Endlagerthematik hinaus.

\section{Danksagung}

Wir danken René König, Christoph Schneider, Katja Baur, Wiebke Rössig, Stefanie B. Seitz und Ulrich Smeddinck für ihre Anmerkungen zu einer früheren Fassung dieses Diskussionsbeitrags.

\section{Anmerkungen}

1) Zur besseren Lesbarkeit wird das generische Maskulinum verwendet.

2) Die zitierten Wortbeiträge sind soweit verfügbar als Video online nachzuhören: https://www.bundestag.de/endlager/mediathek/videos (download 28.9.15)

3) Ein Teilnehmer hatte zuvor kritisiert, der Bürgerdialog suggeriere einen gesellschaftlichen Konsens zum Bau eines Endlagers. Zur Abhängigkeit der Akzeptanz der partizipativen Standortsuche von der Akzeptanz der politischen Handlungsempfehlung für ein Endlager und dessen Kriterien vgl. Grunwald (2010, S. 97) und Grunwald (2015, S. 10).

4) Verweis auf eine mögliche unabhängige Stiftung, Kommission (2016, S. 11f.).

\section{Literatur}

acatech - Deutsche Akademie der Technikwissenschaften (Hg.), 2011: Akzeptanz von Technik und Infrastrukturen. Anmerkungen zu einem aktuellen gesellschaftlichen Problem. Berlin

Becker, J., 2016: Medien im Krieg, Krieg in den Medien. Wiesbaden

Bogner, A., 2010: Partizipation als Laborexperiment. Paradoxien der Laiendeliberation in Technikfragen. In: Zeitschrift für Soziologie 39/2 (2010), S. 87-105

Böschen, S.; Walk, H. (Hg.), 2014: Technik und Protest - Von Stuttgart 21 über Atomkraftendlager bis Fracking und Nanotechnologie. In: Forschungsjournal Soziale Bewegungen 27/4 (2014)

Bröckling, U., 2015: Gute Hirten führen sanft. Über Mediation. In: Mittelweg 36 24/1-2 (2015), S. 171-186

Decker, M.; Fleischer, T., 2014: Bürgerdialoge: „Die Notwendigkeit für solche Formate ist auf jeden Fall gegeben“ - Interview mit Dr. Christoph Braß über die 
Bürgerdialoge „Zukunftstechnologien und Zukunftsthemen" des BMBF. In: Technikfolgenabschätzung Theorie und Praxis 23/2 (2014), S. 56-62

De Hert, P.; Kloza, D.; Wright, D., 2012: Recommendations for a Privacy Impact Assessment Framework for the European Union. Brüssel

Dehmer, D., 2015: Die neue Suche nach einem Endlager. Dialog als Monolog. Tagespiegel vom 21.6.15

Deutschlandradio Kultur, 2015: Deutsche Skepsis. Die Legende von der Technik-Angst. Technikphilosoph Armin Grunwald im Gespräch mit Dieter Kassel. Interview vom 16.3.15

Grunwald, A., 2010: Technikfolgenabschätzung Eine Einführung. Berlin

Grunwald, A., 2015: Akzeptanz. Diskussionsbeitrag Endlager-Kommission AG 1 vom 24.3.15. Unveröff. Manuskript

Grunwald, A., 2016 (i. E.): Welchen Einfluss haben die großen Havarien der Kernenergie auf ihre ethische Beurteilung 2015? In: Quante, M. (Hg.): Geschichte - Gesellschaft - Geltung. XXIII. Deutscher Kongress für Philosophie

Grunwald, A.; Grünwald, R.; Oertel, D. et al., 2002: Kernfusion Sachstandsbericht. TAB-Arbeitsbericht Nr. 75. Berlin

Hennen, L., 1994: Ist die (deutsche) Öffentlichkeit „technikfeindlich“? Ergebnisse der Meinungs- und der Medienforschung. Erster Sachstandsbericht im Rahmen des Monitoringprojektes „Technikakzeptanz und Kontroversen über Technik“. TAB-Arbeitsbericht Nr. 24. Bonn

Kommission Lagerung hoch radioaktiver Abfallstoffe, 2015a: Bürgerdialog „Standortsuche für hochradioaktive Abfallstoffe“. Evaluationsbericht (DIALOGIK/ European Institute for Public Participation) Vorlage am 15.9.15, K-Drs. 117a, K-Drs./AG1-45a

Kommission Lagerung hoch radioaktiver Abfallstoffe, 2015b: Konzept für die Beteiligung der Öffentlichkeit am Bericht der Kommission Lagerung hoch radioaktiver Abfallstoffe. Vorlage am 15.5.15, K-Drs. 107a

Kommission Lagerung hoch radioaktiver Abfallstof$f e$, 2016: Entwurf des Berichtsteils zu Teil B - Kapitel 7 (Ein akzeptiertes Auswahlverfahren). Vorlage der AG 1 für die 22. Sitzung der Kommission am 15.2.16, K-Drs. 180

Kreimeier, K., 2001: Mediengeschichte des Films. In: Schanze, H. (Hg.): Handbuch der Mediengeschichte. Stuttgart, S. 425-454

Kropp, C., 2013: Demokratische Planung der Klimaanpassung? Über die Fallstricke partizipativer Verfahren im expertokratischen Staat. In: Knierim, A.;
Baasch, S.; Gottschick, M. (Hg.): Partizipation und Klimawandel - Ansprüche, Konzepte und Umsetzung. München, S. 55-74

Kühl, Y.; Wittstock, F.; Wulf, N., 2015: Auf Augenhöhe? Beteiligung lernen in der Endlagerdebatte. In: Technikfolgenabschätzung - Theorie und Praxis 24/3 (2015), S. 132-136

Mouffe, C., 2007: Über das Politische. Wider die kosmopolitische Illusion. Frankfurt a. M.

Renn, O.; Zwick, M., 1997: Risiko- und Technikakzeptanz. Berlin

Rossnagel, A.; Ewen, C.; Götz, K. et al., 2014: Mit Interessengegensätzen fair umgehen - zum Einbezug der Öffentlichkeit in Entscheidungsprozesse zu dezentralen Energieanlagen. In: Zeitschrift für Neues Energierecht 18/4 (2014), S. 329-337

Ruesch, M.; Märker, O., 2012: Real Name Policy in E-Participation. The Case of Gütersloh's Second Participatory Budget. In: Parycek, P.; Edelmann, N.; Sachs, M. (Hg.): CeDEM12: Proceedings of the International Conference for E-Democracy and Open Government. Krems, S. 109-123

Seemann, M., 2011: Das Partizipations-TransparenzDilemma. FES-Berliner Positionen 05. Berlin

Sturgis, P., 2014: On the Limits of Public Engagement for the Governance of Emerging Technologies. In: Public Understanding of Science 23/1 (2014), S. 38-42

Wagner, T., 2013: Die Mitmachfalle. Bürgerbeteiligung als Herrschaftsinstrument. Köln

Wilk, M.; Sahler, B. (Hg.), 2014: Strategische Einbindung - von Mediationen, Schlichtungen, Runden Tischen ... und wie Protestbewegungen manipuliert werden. Lich

\section{Kontakt}

Yasmine Kühl

Institut für Technikfolgenabschätzung und

Systemanalyse (ITAS)

Karlsruher Institut für Technologie (KIT)

Karlstraße 11, 76133 Karlsruhe

Tel.: +49 721 608-26876

E-Mail: yasmine.kuehl@kit.edu 